\title{
Révision de la loi sur les produits thérapeutiques: la transparence est le meilleur remède
}

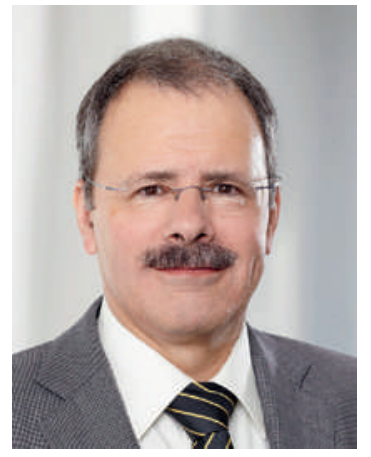

Le Conseil national devrait commencer à examiner la révision de la loi sur les produits thérapeutiques (LPTh) lors de la session de juin. Cette révision poursuit notamment les objectifs suivants: promouvoir les médicaments pédiatriques, simplifier la mise sur le marché des médicaments de la médecine complémentaire, améliorer l'information sur les médicaments et accroître la transparence de ce que l'on nomme les «avantages matériels», c'est-à-dire les bonus et les rabais que les fabricants accordent aux cabinets médicaux, hôpitaux et pharmacies.

La limitation, voire la suppression, de la propharmacie n'est plus à l'ordre du jour et sera traitée ultérieurement. En revanche, la remise, par les pharmaciens, de certains médicaments soumis à ordonnance sans prescription médicale est maintenue, en dépit des réserves émises notamment par la FMH.

\section{Les études sur les médicaments pédiatriques}

et sur l'administration de médicaments chez les personnes âgées doivent être encouragées.

La FMH juge la révision de la LPTh essentiellement positive et salue la volonté d'améliorer la sécurité des patients lors de l'administration de médicaments. Les améliorations proposées dans le domaine de l'information sur les médicaments revêtent également une importance majeure pour la FMH. La disparition de la version imprimée du Compendium des médicaments, au profit d'une version électronique, a en effet pour conséquence une perte d'informations qui peut et qui doit être corrigée dans le cadre de la LPTh. Par ailleurs, la FMH est favorable à l'introduction d'études et à la création d'une banque de données pour les médicaments pédiatriques, et propose d'introduire une réglementation analogue pour l'administration de médicaments chez les personnes âgées. Les médecins disposent en effet de trop peu d'informations étayées par des études pour ce groupe de population en constante progression.

\section{Révision de la LPTh: la FMH soutient l'exigence d'une plus grande transparence.}

La FMH approuve également l'obligation de signaler les intérêts pour les participations économiques dans des entreprises fabriquant ou mettant sur le marché des produits thérapeutiques. La transparence est pour nous une évidence. Quiconque occupe une fonction au sein d'un organe de direction ou de surveillance ou qui exerce une fonction de conseil ou d'expert doit le déclarer. Il en va de même pour toute personne qui bénéficie de rabais lors de l'achat de produits thérapeutiques, qui doit l'indiquer de manière transparente dans ses comptes. Le manque de transparence dans un domaine aussi sensible peut déboucher sur des conflits d'intérêts susceptibles de nuire à une utilisation sûre des médicaments et de compromettre la confiance entre médecin et patient et par là même la crédibilité de l'ensemble de notre profession.

Nous devons également faire preuve de transparence dans notre façon de gérer les avantages. Dans ce domaine, il convient de clarifier ouvertement le rôle et les intérêts de chacun: assureurs, patients, médecins, hôpitaux et industrie. La FMH est prête à relever ce défi.

Dr Jürg Schlup, président de la FMH 